\title{
ALMOST COMMUTATIVE BANDS
}

\author{
by T. E. HALL $\dagger$ \\ (Received 15 January, 1971)
}

1. Introduction. To find a "description of the structure of bands which is complete modulo semilattices" (from page 25 of [1]) seems to be a very difficult problem. As far as the author is aware, the only class of bands (except for rectangular bands) for which this problem has been solved (see [4] and [3]) is the class of all bands satisfying a generalization of commutativity, namely the condition that $e f g h=e g f h$ for all elements $e, f, g$ and $h$.

The purpose of this paper is to give a solution to this problem for a further class of bands, which we call the class of almost commutative bands: a band is called almost commutative if any pair of elements are either $\mathscr{F}$-related or commute. It is easily seen from [1, Theorem 4.6] that a band $B$ is almost commutative if and only if, for all $e, f \in B$, either $e f=f e$ or both $e f e=e$ and $f e f=f$.

Three examples of almost commutative bands played a major role in $[2, \S 4]$ in the solution of two problems posed in [6]. The author was helped in the writing of this paper by an expository thesis of J. Pippey [5], which included the results given here.

2. Preliminaries. We use wherever possible, and usually without comment, the notations of Clifford and Preston [1].

Let $B$ be any band. Then from [1, Theorem 4.6] $B$ is a semilattice of rectangular bands; that is to say, for some semilattice $Y$ and rectangular subbands $\left\{E_{\alpha}: \alpha \in Y\right\}$ of $B, B=\bigcup_{\alpha \in Y} E_{\alpha}$, and for all $\alpha, \beta \in Y, E_{\alpha} \cap E_{\beta}=\square$ if $\alpha \neq \beta$, and $E_{\alpha} E_{\beta} \subseteq E_{\alpha \beta}$; further, each $E_{\alpha}$ is a $\mathscr{J}$-class of $B$. If $e \in E_{\alpha}$, then we shall sometimes denote $E_{\alpha}$ by $E(e)$. It is clear that for any $e, f \in B$

$$
e E(e f) e=\{g \in E(e f): g \leqq e\},
$$

where as usual $g \leqq e$ means $e g=g=g e$.

\section{Almost commutative bands.}

LEMMA 1. For any elements $e, f$ in the band $B$,

$$
|[e E(e f) e] \cap[f E(e f) f]| \leqq 1 .
$$

Proof. Suppose that the set $[e E(e f) e] \cap[f E(e f) f] \neq \square$ and take any element $g$ of this set. Then $g \leqq e$ and $g \leqq f$, from which we easily see that $g \leqq e f$. But $e f, g \in E(e f)$, a rectangular band; so $g=e f$. Therefore the set above contains at most one element, namely ef (further, we may easily see that it contains $e f$ if and only if $e f=f e$ ).

LEMMA 2. The band $B$ is almost commutative if and only if, for any $\alpha, \beta \in Y$ and any $e \in E_{\alpha}, f \in E_{\beta}, \beta<\alpha$ implies $f<e$.

$\dagger$ This research was supported by a Nuffield Travelling Fellowship. 
Proof. Suppose that $B$ is almost commutative and take any $\alpha, \beta \in Y$ such that $\beta<\alpha$. Then, for any $e \in E_{\alpha}, f \in E_{\beta}$, we have $e f=f e \in E_{\alpha \beta}=E_{\beta}$. But, since $e f=f e$, we see that $e f \leqq f$, whence $e f=f$, since $E_{\beta}$ is a rectangular band. Thus $f<e$.

Conversely suppose, for all $\alpha, \beta \in Y$ and for all $e \in E_{\alpha}, f \in E_{\beta}$, that $\beta<\alpha$ implies $f<e$. Take any elements $g, h \in B$ such that $J_{g} \neq J_{h}$, i.e. $E(g) \neq E(h)$. Then $g \in E_{\gamma}$ and $h \in E_{\delta}$ for some $\gamma, \delta \in Y$ with $\gamma \neq \delta$. If $\gamma<\delta$, then $g<h$, whence $g h=g=h g$ and, similarly, if $\delta<\gamma$, then $g h=h=h g$. Let us consider then the remaining case, namely when $\gamma$ and $\delta$ are not comparable; then $\gamma \delta<\gamma$ and $\gamma \delta<\delta$. Since $g h, h g \in E_{\gamma \delta}$, we have that both $g h$ and $h g$ are less than both $g$ and $h$; so, from Lemma $1, g h=h g$. Thus $B$ is almost commutative.

Remark 1. Lemma 2 contrasts with the case when $B$ satisfies $e f g h=e g f h$ for all $e, f, g$, $h \in B$, for this is true of $B$ if and only if $\left|e E_{\beta} e\right|=1$ for all $\beta \in Y, e \in B$ [5, Theorem 6].

From Lemmas 1 and 2 we see that, if $B$ is almost commutative, then, for any $\alpha, \beta \in Y$, $\alpha \neq \alpha \beta \neq \beta$ implies $\left|E_{\alpha \beta}\right|=1$. We have thus proved already the final statement of the following theorem.

THEOREM 1. Let now $Y$ be any semilattice and let $\left\{E_{\alpha}: \alpha \in Y\right\}$ be any set of pairwise disjoint rectangular bands such that $\left|E_{\alpha}\right|=1$ if $\alpha=\beta \gamma$ for some $\beta, \gamma \in Y$ and $\beta \neq \alpha \neq \gamma$; if this is the case, then let $e_{\alpha}$ denote the only element of $E_{\alpha}$. Let the multiplication in each $E_{\alpha}$ be denoted by juxtaposition. Put $B=\bigcup_{\alpha \in Y} E_{\alpha}$ and define a multiplication $\circ$ for $B$ as follows: for any $\alpha, \beta \in Y$ and for any $e \in E_{\alpha}, f \in E_{\beta}$, define

$$
e \circ f=\left\{\begin{array}{l}
e \text { if } \alpha<\beta, \\
e f \text { as in } E_{\alpha} \text { if } \alpha=\beta, \\
f \text { if } \alpha>\beta, \\
e_{\alpha \beta} \text { if } \alpha \neq \alpha \beta \neq \beta .
\end{array}\right.
$$

Then $B$ is an almost commutative band. Conversely any almost commutative band is obtained in this way.

Proof. To show that $\circ$ is associative we shall only assume that the $E_{\alpha}(\alpha \in Y)$ are semigroups and not necessarily rectangular bands.

Take any $e, f, g \in B$. Then $e \in E_{\alpha}, f \in E_{\beta}$ and $g \in E_{\gamma}$ for some $\alpha, \beta, \gamma \in Y$. It is clear from the definition of $\circ$ that $e \circ f \in E_{\alpha \beta},(e \circ f) \circ g \in E_{\alpha \beta \gamma}$ and $e \circ(f \circ g) \in E_{\alpha \beta \gamma}$. Hence, if $\left|E_{\alpha \beta \gamma}\right|=1$, then $(e \circ f) \circ g=e \circ(f \circ g)$.

Suppose then that $\left|E_{\alpha \beta \gamma}\right|>1$. Then $\alpha \beta$ and $\gamma$ are comparable.

Case 1: $\gamma<\alpha \beta$. Then $(e \circ f) \circ g=g$ and, since $\gamma<\beta$ and $\gamma<\alpha, e \circ(f \circ g)=e \circ g=g$, giving $(e \circ f) \circ g=e \circ(f \circ g)$.

Case 2: $\gamma=\alpha \beta$. Then $\left|E_{\alpha \beta}\right|=\left|E_{\alpha \beta \gamma}\right|>1$, whence $\alpha$ and $\beta$ are comparable.

Case 2(a): $\alpha<\beta$. Then $\gamma=\alpha \beta=\alpha<\beta$, whence

$$
(e \circ f) \circ g=e \circ g=e \circ(f \circ g) \text {. }
$$


Case 2(b): $\alpha=\beta$. Then $\alpha=\beta=\gamma$ and clearly $(e \circ f) \circ g=e \circ(f \circ g)$.

Case 2(c): $\alpha>\beta$. Then $\alpha>\beta=\alpha \beta=\gamma$, whence

$$
(e \circ f) \circ g=f \circ g=f g=e \circ(f g)=e \circ(f \circ g) .
$$

Case 3: $\gamma>\alpha \beta$. Once again $\left|E_{\alpha \beta}\right|=\left|E_{\alpha \beta \gamma}\right|>1$, whence $\alpha$ and $\beta$ are comparable.

Case 3(a): $\alpha<\beta$. Then $\alpha=\alpha \beta<\gamma$ and $(e \circ f) \circ g=e \circ g=e$. Also $\alpha \leqq \beta \gamma$. If $\alpha=\beta \gamma$, then $\beta \neq \beta \gamma \neq \gamma$, giving $\left|E_{\alpha \beta \gamma}\right|=\left|E_{\beta \gamma}\right|=1$, a contradiction. Hence $\alpha<\beta \gamma$, giving $e \circ(f \circ g)=$ $e=(e \circ f) \circ g$.

Case 3(b): $\alpha=\beta$. Then $\beta=\alpha \beta<\gamma$, giving

$$
(e \circ f) \circ g=e \circ f=e \circ(f \circ g) \text {. }
$$

Case 3(c): $\alpha>\beta$. Then $\beta=\alpha \beta<\gamma$ and

$$
(e \circ f) \circ g=f \circ g=f=e \circ f=e \circ(f \circ g) \text {. }
$$

We now have that $\circ$ is associative, and clearly then $B$ is an almost commutative band.

\section{REFERENCES}

1. A. H. Clifford and G. B. Preston, The algebraic theory of semigroups, Amer. Math. Soc. Mathematical Surveys, No 7, Vol. I (Providence, R.I., 1961).

2. T. E. Hall, Orthodox semigroups, Pacific J. Math. 39 (1971), 677-686.

3. J. M. Howie, Naturally ordered bands, Glasgow Math. J. 8 (1967), 55-58.

4. N. Kimura and M. Yamada, Note on idempotent semigroups II, Proc. Japan Acad. 34 (1958), $110-112$.

5. J. Pippey, Some structure theorems for bands, Honours year thesis (1969), Monash University.

6. M. Yamada, On a regular semigroup in which the idempotents form a band, Pacific J. Math. 33 (1970), 261-272.

UNIVERSITY OF STIRLING 